\title{
Measurement of Thermophysical Properties of Ceramics by the Flash Method
}

\author{
Cláudio S. C. Pinto ${ }^{1 *}$, Henrique Massard ${ }^{1}$, Paulo Couto ${ }^{1}$, Helcio R. B. Orlande ${ }^{1}$, Renato M. \\ Cotta $^{1}$ and Maria C. R. Ambrosio ${ }^{2}$ \\ ${ }^{1}$ Federal University of Rio de Janeiro - UFRJ; Department of Mechanical Engineering - POLI/COPPE; P.O. Box: \\ 68503; Cidade Universitária; 21945-970; Rio de Janeiro - RJ - Brasil. ${ }^{2}$ Federal University of Rio de Janeiro - \\ UFRJ; Department of Metalurgical Engineering - POLI/COPPE; P. O. Box: 68503, Cidade Universitária; 21945- \\ 970; Rio de Janeiro - RJ - Brasil
}

\begin{abstract}
The flash method, proposed by Parker, Butler, Jenkins and Abbott from the U.S. Navy Radiological Defense Laboratory in 1961, is the most popular method for measuring the thermal diffusivity of solids. In this method, the front surface of a small sample is subjected to a very short burst of radiant thermal energy. The resulting temperature rise on the opposite surface of the sample is measured and the thermal diffusivity is computed from the temperature rise versus time data. Also, the specific heat can be computed from the measured data, thus allowing for the calculation of the thermal conductivity. Several theoretical models are available for the flash method, which include adiabatic boundary conditions, heat losses, surface coating effects, among other aspects. In this paper, tests were made for the identification of thermo-physical properties of a Ceramic block. The Netzsch Nanoflash LFA 447/1 of LTTC/COPPE/UFRJ was used for the measurements.
\end{abstract}

Key words: Flash Method, Thermophysical Property, Ceramic block

\section{INTRODUCTION}

The second half of the twentieth century has seen the subject of thermal properties measurement changing from an exercise mostly of academic interest to a complex issue attracting the attention of a large number of people in different sectors of industry, government and universities. The explosion in materials development for many emerging technologies has produced substantial quantities of improved materials and composites in different forms. This has led to a corresponding requirement for evaluation of their thermophysical properties more rapidly and often, under conditions that cannot be satisfied using the appropriate classical methods [1]. These classical methods, which involved the fitting of steady state and non-steady state experimental temperature data to theoretical models, were usually time consuming. Additionally, the large size of the samples imposed intolerable limitations, usually tied to heat losses and contact resistance between the specimen, and its associated heat sources, heat sinks and measurement devices. The Flash Method, first proposed by Parker et al. [2], eliminated the problem of contact resistance of the classical methods, and minimized the heat losses by making the measurement time short enough so that very little cooling can take place. This method consists of heating the front surface of a thermally

\footnotetext{
${ }^{*}$ Author for correspondence
} 
insulated specimen with a high-intensity shortduration radiant heat pulse and measuring the temperature evolution on the back surface by the use of an infrared (IR) detector. The non-intrusive backside measurement method eliminates the concern and issues with sensor attachment to the sample, and removes all uncertainties associated with contact resistance and sensor measurement accuracy.

Although different industries and research institutes are already using the Flash Method for thermal properties measurements in Brazil, the lack of a national standardized procedure does not allow the inter-laboratory comparison of thermal properties, which reduces the measurement traceability. Therefore, the National Institute of Metrology, Standardization and Industrial Quality (INMETRO) in a joint project with the Federal University of Rio de Janeiro (COPPE/UFRJ), funded by the National Council for Scientific and Technological Development (CNPq), is working towards the implementation of a primary method for the measurement of the thermal diffusivity of solids materials based on the Flash Method, according to the ASTM standard E1461-0 [3]. This standardized primary method will stimulate the development of new techniques for thermophysical properties identifications, which is of main interest of several Brazilian industries, decreasing its external dependence on qualified materials testing and thermal characterization. This paper describes de determination of thermal diffusivity, heat capacity and thermal conductivity of a high porosity ceramic block developed for civil construction. Ceramic blocks are a good alternative to improve thermal comfort and for the reduction of electricity consumption [4].

\section{MATHEMATICAL MODELS}

Below, we present the models available for the identification of thermal diffusivity with the Flash Method.

In 1961, Parker et al. [2] published their pioneering work on the development of a method for the thermal diffusivity identification of solid materials. Although not specifically devised to, the proposed method also permits the identification of the specific heat of materials.

In the method developed by Parker et al. [2], a small and thin specimen is subjected to a highintensity short-duration radiant energy pulse. The energy of the pulse is absorbed on the front surface of the specimen and the resulting rear surface temperature rise is recorded, as illustrated in Fig. (1). Parker et al. [2] calculated the thermal diffusivity value from the specimen thickness and from the time required for the rear surface temperature rise to reach $50 \%$ of its maximum value $\left(\mathrm{t}_{0,5}\right)$. Generally, the temperature rise in the specimen is small, so that the physical properties can be assumed constant during the test. Therefore, if the thermal diffusivity of the specimen is to be determined over a temperature range, the test procedure must be repeated at each temperature of interest. Parker et al. [2] named their test procedure as the Flash Method. Such a method is particularly advantageous because of the simple specimen geometry, small specimen size requirements, rapidity of measurement and handling, with a single apparatus, of materials having a wide range of thermal diffusivity values over a large temperature range.

In order to obtain the thermal diffusivity from $t_{0,5}$, Parker et al. [2] used a one-dimensional heat conduction model, neglecting heat losses and assuming that the energy input was instantly deposited within a small depth of the specimen. In order to cope with experimental conditions where heat losses cannot be neglected and with a finite pulse duration, other authors proposed alternative mathematical models for the heat conduction problem in the specimen, such as those described in Cowan [5, 6]; Cape and Lehman (1963) [7]; and Clark and Taylor (1975) [8]. In fact, the finite pulse width effect occurs strongly when thin samples of high thermal diffusivity are tested, while heat losses become dominant at high temperatures when testing thick samples [3]. Recently, more involved models dealing with the coupled conduction-radiation heat transfer within the specimen were proposed for semi-transparent materials $[9,10]$.

\section{EXPERIMENTAL APPARATUS AND ANALISYS OF EXPERIMENTAL DATA}

In this section, the technical characteristics of the equipment used for the identification of the thermo-physical properties of a ceramic block are discussed. The Netzsch Nanoflash LFA 447/1 is a tabletop instrument that works with a high power Xenon-Flash lamp in the temperature range of 
room temperature up to $200^{\circ} \mathrm{C}$, and it has an integrated sample changer for 4 samples. Figures 2 and 3 show a sketch of the instrument systems. The high-power Xenon flash lamp, which is surrounded by a parabolic mirror (reflector), is able to supply a radiant pulse energy up to $10 \mathrm{~J}$ (up to $5 \mathrm{~J} / \mathrm{cm}^{2}$ ), selectable by the measurement software, in the wavelength range of $150 \mathrm{~nm}$ to $2000 \mathrm{~nm}$. The software also allows the energy pulse length to be adjusted to $0.1,0.2$ or $0.4 \mathrm{~ms}$.

The integrated sample changer allows up to 4 different sample positions. Each sample is moved automatically into the measurement position. The measurement software controls the sample positioning. The integrated sample changer uses interchangeable sample holders for rounded ( $\square 10$ $\mathrm{mm}, \square 12.7 \mathrm{~mm}$ and $\square 25.4 \mathrm{~mm}$ ) or squared samples $(10 \mathrm{~mm} \times 10 \mathrm{~mm}$ and $8 \mathrm{~mm} \times 8 \mathrm{~mm})$. The sample changer is surrounded by glass windows for protection of the flash lamp and of the IR detector.

The LFA 447/1 uses an InSb-IR detector in the wave-length range of 2000 to $5000 \mathrm{~nm}$ cooled by liquid ni-trogen, which allows a non-contact fast measurement of the temperature rise in the back of the sample. The close connection to the preamplifier system permits a fast data acquisition $(500 \mathrm{kHz}, 12 \mathrm{Bit})$ and a measurement of 2000 points per shot (detector signal). An integrated furnace (heater) maintains the sample tem-perature stable during measurement. The heater is integrated in the automatic sample changer, which has a low thermal mass allowing fast heating/cooling rates. Sample temperature measurement (thermocouple) is realized in the sample carrier. A Julabo Chiller is used to cool the surrounding components.

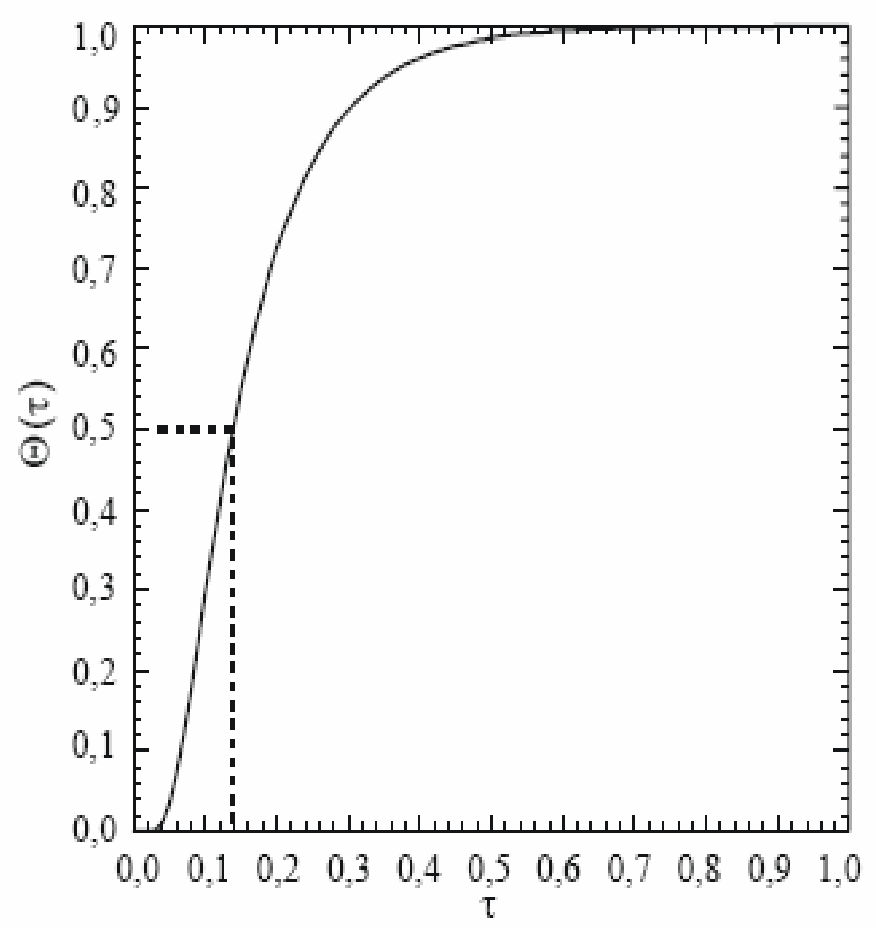

Figure 1 - Thermal diffusivity measurement according to Parker et al (1961) [2].

The LFA $447 / 1$ is capable of thermal diffusivity measurements in the range of $0.01 \mathrm{~mm}^{2} / \mathrm{s}$ up to $1000 \mathrm{~mm}^{2} / \mathrm{s}$, with an accuracy of $3-5 \%$ for most materials. The specific heat accuracy is $5-7 \%$. This allows the calculation of the thermal conductivity in the range of $0.1 \mathrm{~W} / \mathrm{m} . \mathrm{K}$ to $2000 \mathrm{~W} / \mathrm{m} . \mathrm{K}$ with an accuracy of $3-7 \%$ for most materials.

The analysis of the experimental data is performed with Proteus software, provided by Netzsch, which includes different mathematical models, such as: Adiabatic (Parker et al.) [2], Cowan [5, 6], 
Cape-Lehmann [7], and Radiation (Mehling et al.) [9].

One of the experimental parameters that strongly influences the diffusity identification is the finite pulse duration compared to the heat diffusion time. Actually, the laser beam has a short but finite pulse duration. The intensity distribution of the beam within the pulse duration is normally at maximum. This pulse intensity function can influence the duration of the temperature distribution inside the test piece. If this pulse duration is long, compared to heat transport time that is needed to reach $50 \%$ of the maximum temperature change at the back of the test piece, then this effect has to be taken into account when determining the thermal diffusivity. In NetzschLFA software, this pulse correction can be taken also into account according to Lechner and Hahne [11]. If the pulse duration is comparable to the necessary time for heating the back of the test piece, one has to carry out a correction of the finite pulse width. The used mathematical model in the Netzsch-LFA software is the following approximation of a laser-pulse [11]:

where $\tau_{1}, \tau_{2}$, and $\tau_{3}$, are time constants automatically determined to fit the pulse, and delay is the time delay between the beginning of the measurement and the beginning of the pulse shot, automatically determined by the software. The rise of the laser impulse is deter-mined by the time constant $\tau_{1}$, the following slow decay by the time constant $\tau_{2}$. The parameter $t_{\text {end }}$ determines the point in time of the fast decay, which takes place with the time constant $\tau_{3}$.

\section{RESULTS AND DISCUSSION}

The LFA 447/1 analysis software was used for the identification of the thermophysical properties of a ceramic block.

Porous blocks were produced from a mixture of caulinitic clays and conformed by extrusion. Next, the blocks were dried and sintered between $900^{\circ} \mathrm{C}$ and $1100^{\circ} \mathrm{C}$. One square sample was manufactured for testing. The sample surfaces are flat and parallel to provide an accurate measurement of the sample thickness, and to improve the one- dimensional heat transfer characteristic of the tests. To determine the sample thickness, a micrometer was used, and measurements were performed to at least three significant figures, at four different points on the sample. The average value of the four measurements was considered as the thickness value for the tests.

Prior the measurements, the samples were coated with a graphite spray in order to enhance the absorption of the Xenon light pulse energy and the emission of IR radiation to the temperature detector. The graphite coating greatly increases the resulting signal-to-noise ratio when the surface is highly reflective, as with metals or ceramics with a metal film. The sample mass was measured with a precision scale. The bulk density of the sample was calculated by dividing the sample mass by the apparent sample volume calculated from the measured sample dimensions.

A Pyrex specimen was used as a reference sample for the specific heat estimation of the ceramic block. Both reference and test samples were coated side-by-side and at the same time to provide a similar coating thickness. Table 1 shows the dimensions and densities of the test and reference samples used in this investigation.

The Pyrex reference sample and the ceramic test samples were loaded into the sample holder and tested between $25^{\circ}$ up to $175^{\circ} \mathrm{C}$, with a $25^{\circ} \mathrm{C}$ temperature step. A total of 2 measurements were performed at each temperature level in the test sample and in the reference sample. Figure 4 shows an example of the experimental raw data (dots) for the temperature rise at the rear surface of the ceramic block obtained with the LFA 447-1 Flash Method apparatus. The continuous line corresponds to the model used for the thermal diffusivity identification. The model was least square fitted to the raw data.

Figures 5 shows the experimental results for the thermal diffusivity measurement of the ceramic block ac-cording to the LFA 447/1 Nanoflash analysis software. Table 2 shows the numerical value for the thermal diffusivity. The standard deviation and the correlation coefficient between the temperature signal (raw data) and the model used to fit the data (see Fig. 4) are also presented in Tab. 2. The correlation coefficient is used to determine the best mathematical model for the thermal diffusivity calculation. Generally, the best mathematical model for the tests conducted was that of Radiation with pulse correction, even though all the methods had a coefficient very close 
to 1. The conductivity and specific heat experimental data are presented in Figs. 6 and 7, with the standard deviation of the experimental results shown in Tab. 3 and 4.

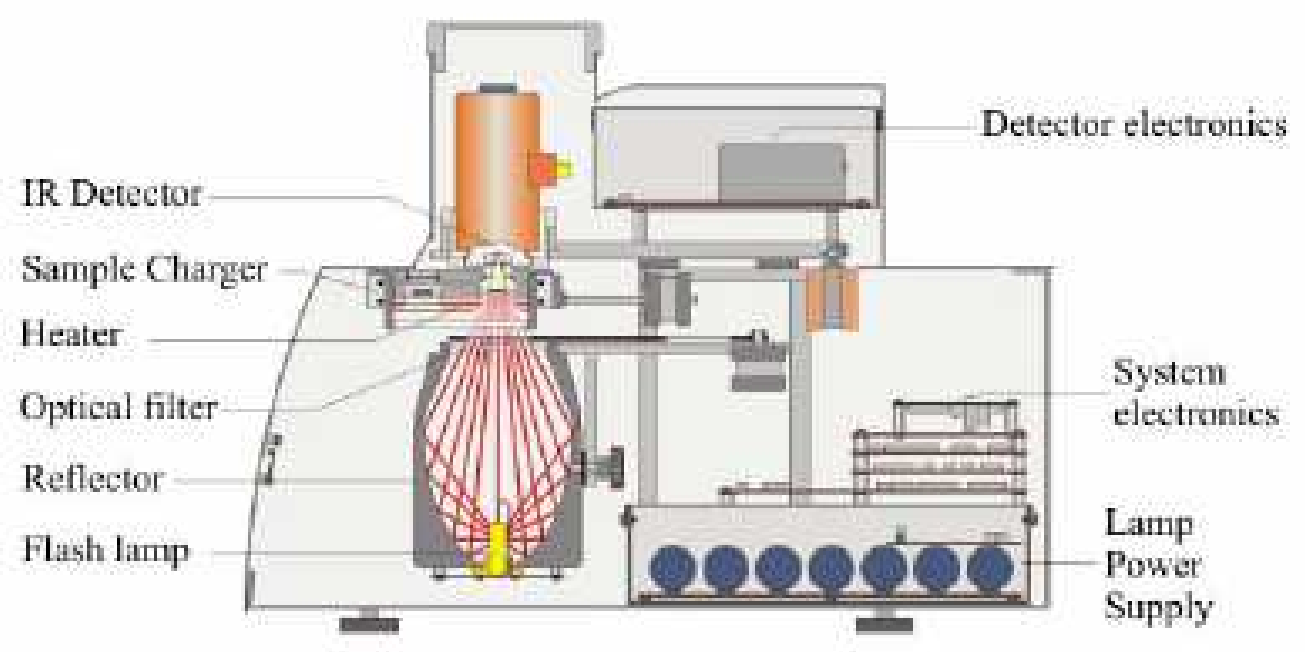

Figure 2 - Schematics of the Netzsch Nanoflash LFA 447/1.

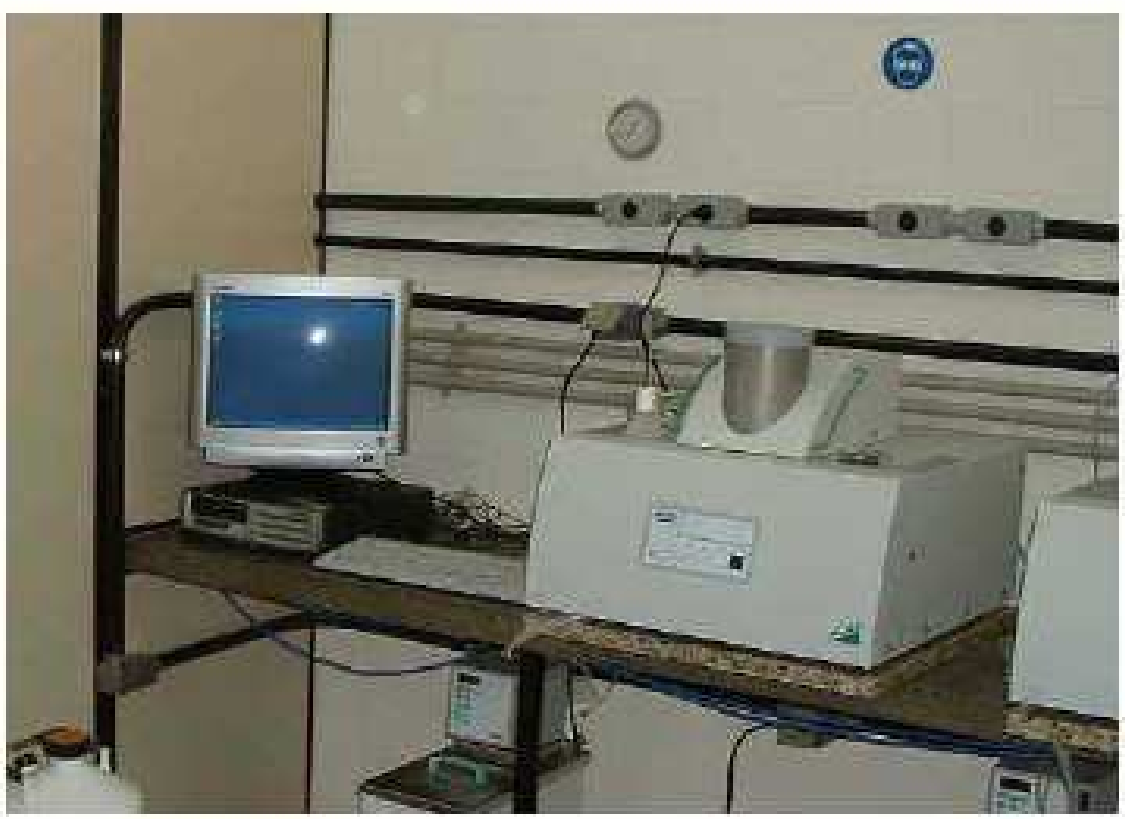

Figure 3 - Thermal Metrology Unit Prof. Roberto de Souza - UNIMET (COPPE / UFRJ) 
Table 1 - Samples dimensions and densities

\begin{tabular}{c|c|c|c}
\hline Sample & Side $[\mathbf{m m}]$ & Thickness [mm] & Density [g/cm ] \\
\hline Ceramic & 9,99 & 3,00 & 1,69 \\
Pyrex & 9,95 & 2,976 & 2,22 \\
\hline
\end{tabular}

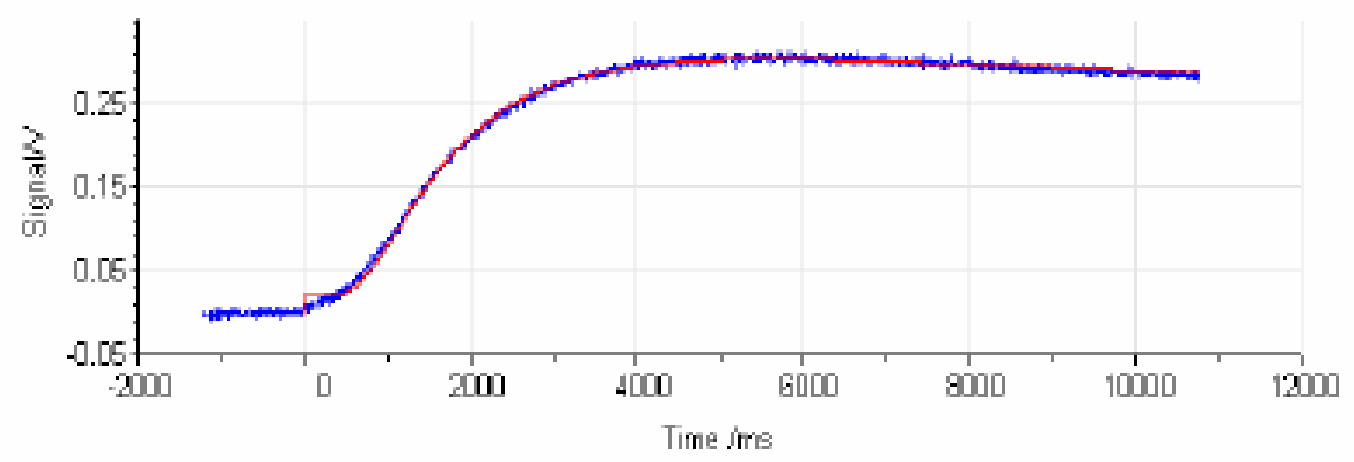

Figure 4 - Histogram of the rear face.

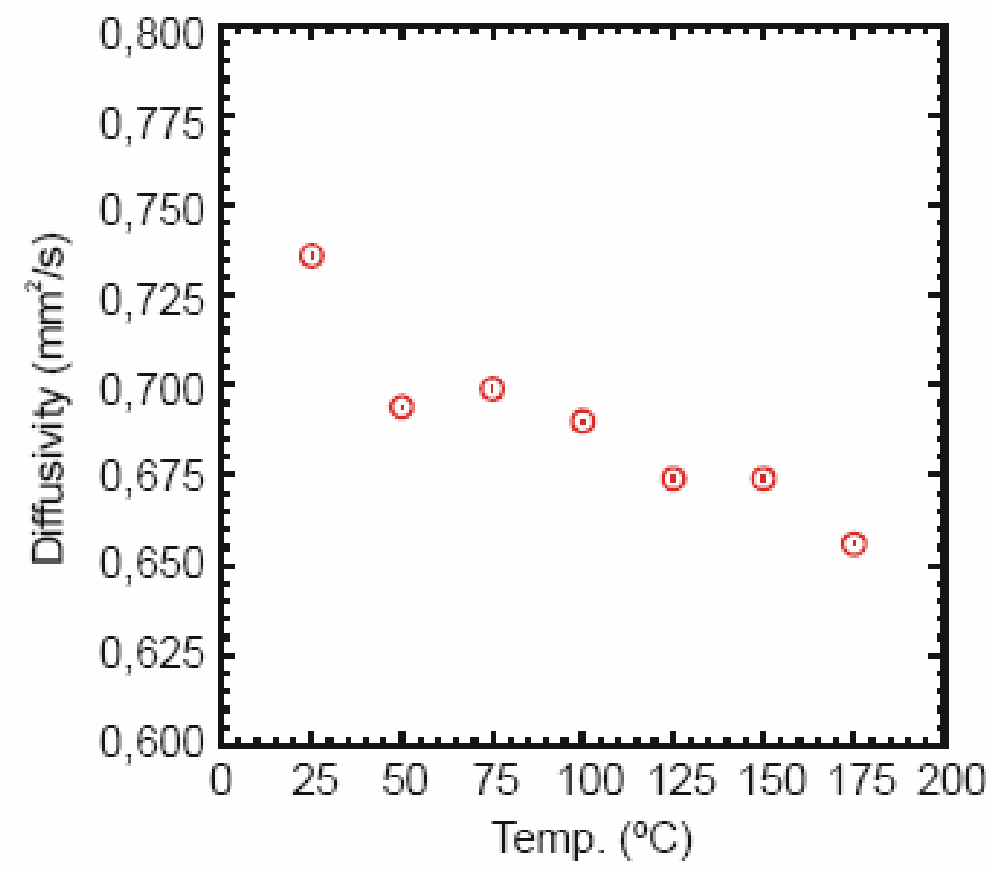

Figure 5 - Thermal diffusivity of ceramic 
Table 2 - Thermal diffusivity, standard deviation and correlation coefficients.

\begin{tabular}{c|c|c|c}
\hline Temp. ( $\left.^{\mathbf{C}}\right)$ & Thermal Diffusivity $\left(\mathbf{m m}^{\mathbf{2}} / \mathbf{s}\right)$ & Std. Deviation $\left(\mathbf{m m}^{\mathbf{2}} / \mathbf{s}\right)$ & Correlation Coefficient \\
\hline 25 & 0.736 & 0.010 & 0.999 \\
50 & 0.694 & 0.019 & 0.999 \\
75 & 0.699 & 0.009 & 0.999 \\
100 & 0.690 & 0.009 & 0.999 \\
125 & 0.674 & 0.011 & 0.999 \\
150 & 0.674 & 0.008 & 0.999 \\
175 & 0.656 & 0.018 & 0.999 \\
\hline
\end{tabular}

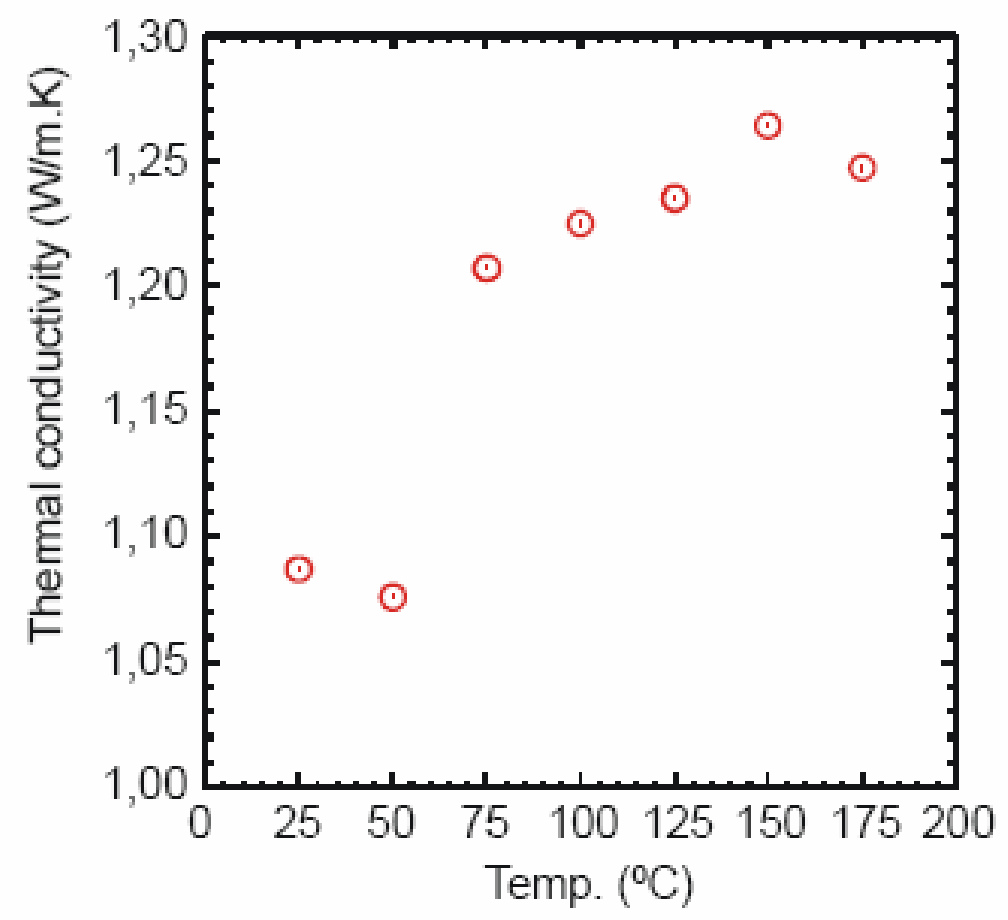

Figure 6 - Thermal conductivity of ceramic

Table 3 - Thermal conductivity and standard deviations.

\begin{tabular}{c|c|c}
\hline Temp. $(\mathbf{0})$ & Thermal Conductivity $(\mathbf{W} / \mathbf{m} * \mathbf{K})$ & Std Deviation $(\mathbf{W} / \mathbf{m} * \mathbf{K})$ \\
\hline 25 & 1.087 & 0.015 \\
50 & 1.076 & 0.031 \\
75 & 1.207 & 0.015 \\
100 & 1.225 & 0.016 \\
125 & 1.235 & 0.020 \\
150 & 1.264 & 0.014 \\
175 & 1.247 & 0.035 \\
\hline
\end{tabular}




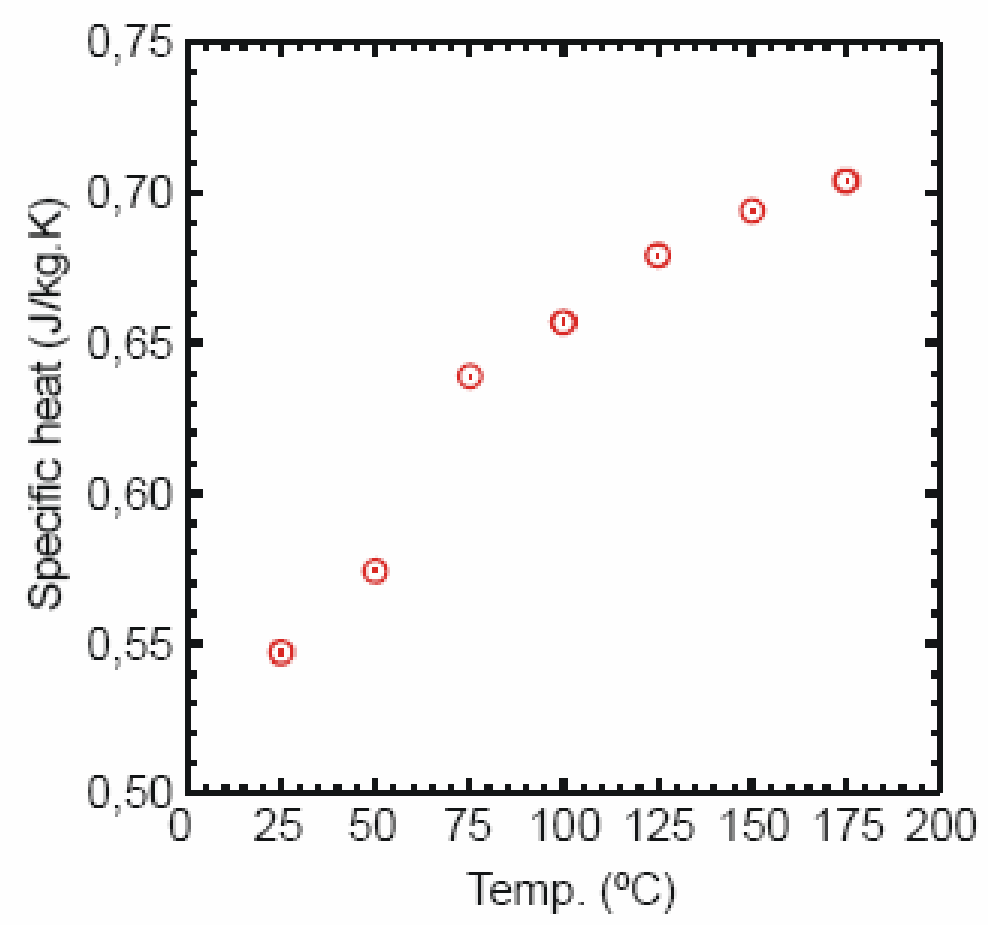

Figure 7 - Specific heat of ceramic.

Table 4 - Specific heat and standard deviations.

\begin{tabular}{c|c|c}
\hline Temp. $(\mathbf{0} \mathbf{C})$ & Specific Heat $(\mathbf{J} / \mathbf{g} / \mathbf{K})$ & Std. Deviation $(\mathbf{J} / \mathbf{g} / \mathbf{K})$ \\
\hline 25 & 0.547 & 0.018 \\
50 & 0.574 & 0.023 \\
75 & 0.639 & 0.027 \\
100 & 0.657 & 0.01 \\
125 & 0.679 & 0.014 \\
150 & 0.694 & 0.025 \\
175 & 0.704 & 0.044 \\
\hline
\end{tabular}

The value of the thermal conductivity is in great agreement with the value found with the guarded hot plate method. The value found with the hot plate was $0.99 \mathrm{~W} / \mathrm{K} . \mathrm{m}\left(40^{\circ} \mathrm{C}\right)$ and the flash method value was $1.076 \mathrm{~W} / \mathrm{K} . \mathrm{m}\left(50^{\circ} \mathrm{C}\right)$ [4].

\section{CONCLUSIONS}

For the ceramic block studied, within the temperature range of the tests conducted in this work, no significant difference was observed on the Thermophysical properties. This shows that the ceramic has little variation of the thermophysical properties with temperature. The values for thermal diffusivity obtained here are in ex-cellent agreement with those available in the literature. The best mathematical model for this material is that of radiation with pulse correction.

\section{ACKNOWLEDGEMENTS}

This work is supported by CNPq through the grant 460.203/01-0. 


\section{REFERENCES}

[1] Campbell, R. C. and Smith, S. E. (2002), Flash diffusivity method: a survey of capabilities. Electronics Cooling, 8, : (2).

[2] Parker, W. J.; Jenkins, W. J.; Butler, C. P. and Abbott, G. L. (1961), Flash method of determining thermal diffusivity, heat capacity and thermal conductivity. J. App. Physics, 32 : (9), 1679-1684.

[3] ASTM Test Method E1461-01 (2001), Standard test method for thermal diffusivity of solids by the flash method. American Society for Testing and Materials. Annual Book of ASTM Standards. v. 14.02 .

[4] Ambrosio, M. C. R. (2004), Blocos ceramicos de alta porosidade. In: Congresso Nacional de Engenharia Mecanica, 3., Belém. Anais... Belém, PA, Brasil.

[5] Cowan, R. D. (1961), Proposed method of measuring thermal diffusivity at high temperatures. J. App. Physics, 32 : (7), 13631370.

[6] Cowan, R. D. (1963), Pulse method of measuring thermal diffusivity at high temperatures. J. App. Physics, 34 : (4 - part 1), 926 - 927.

[7] Cape J. and Lehman, G. (1963), Temperature and finite pulse-time effects in the flash method for measuring thermal diffusivity. J. App. Physics, 34 : (7), 1909-1913.

[8] Clark, L. M. and Taylor, R. E. (1975), Radiation loss in the flash method for thermal diffusivity. J. App. Physics, 46 : (2), 714-719.

[9] Mehling, H.; Hautzinger, G.; Nilsson, O.; Fricke, J.; Hofmann, R. and Hahn, O. (1998), Thermal diffusivity of semitransparent materials determined by the laser-flash method applying a new analytical model. Int. J. Thermophysics, 19 : (3), 941-949.

[10] Lazard, M.; André, S. and Maillet, D. (2004), Diffusivity measurement of semi-transparent media: model of the coupled transient heat transfer and experiments on glass, silica glass and zinc selenide. Int. J. Heat Mass Transfer, 47, 477-487.

[11] Lechner, T. and Hahne, E. (1993), Finite pulse time effects in flash diffusivity measurements. Thermodynamica Acta, 218, 341-350.

Received: July 29, 2005; Revised: September 05, 2005; Accepted: November 22, 2005. 


\section{FOLHA}

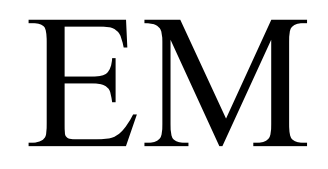

BRANCO 